\title{
Data Thinking: A Canvas for Data-Driven Ideation Workshops
}

\author{
Tizian Kronsbein \\ Berlin School of Economics and Law \\ tizian.kronsbein@gmail.com
}

\author{
Roland M. Mueller \\ Berlin School of Economics and Law \\ roland.mueller@hwr-berlin.de
}

\begin{abstract}
New products and services increasingly follow a data-driven strategy, creating the need for designers, product developers, and teams of individuals to develop products and services with data in mind. This paper provides a data-informed ontology for visual collaboration tools. It presents a prototype of a canvas that could be used during data-oriented design thinking workshops. Using action design research, the Data Innovation Board is tested through iterative cycles of building, intervention, and evaluation and the results are analyzed using triangulation. The suggested datainformed ontology and the proposed canvas facilitate the development of data-driven products and services. The canvas helps teams sharpen their perspective on data challenges from the start and presents a more holistic view on data projects.
\end{abstract}

\section{Introduction}

In 2013, the TV series House of Cards launched on the streaming-service Netflix. It became an international success leading to 2 million new subscribers [33]. Using analytics of viewers' preferences, Netflix's service designers found several trends that ensured them that users would love plot twists, particular characters, and other aspects, even before the show was produced [2].

In 2016, the Washington Post introduced Heliograf, a prototype of an AI-powered bot. It was able to create automated newspaper articles similar to the ones written by humans [18].

In 2018, Google launched Duplex, an AIempowered voice assistant that is able to book appointments in hair salons or restaurants by using speech recognition and text-to-speech synthesis [21].

Among many others, the three examples illustrate that we were able to experience technological breakthroughs in many different domains in the last years. We have seen vast advancements in machine and deep learning, speech recognition, natural language processing, and image recognition. While they all facilitate a variety of different interfaces in versatile products and services, they all have something in common: they are all heavily driven by data. Hence, companies need their employees to understand datadriven products and services better. Harris [14] claimed that companies need to make sure that their employees are able to read, understand, and interpret data. More specifically, employees need to understand how the data strategy is evolving during the different stages of the user journey and the respective product life cycle.

Several studies [25, 30] showed that many companies are still facing data challenges to manage the digitization. McAfee et al. [25] argue that these data challenges are related to people and how they interact with technology, including leadership, talent management, and decision-making. Nevertheless, Frick [11] claimed that in most cases it is not necessary to become sophisticated in math. Data literacy is more about the "continuous learning journey that creates the ability to identify, understand, interpret, create, communicate, and compute pieces of information [...] to develop knowledge and the ability to participate fully in our society" [32]. Similarly, getting practical with data is often more valuable than revising statistical concepts. Bhargava and D'Ignazio [4] claimed that there is a "lack of consistent and appropriate approaches for helping novices learn to 'speak data"'. Most of the tools are designed to help users but are not beginner-friendly [4].

Design Thinking is a method that facilitates userdriven innovation processes [5, 26, 34]. However, data innovation has its own particular challenges. Therefore, this paper seeks to bridge the two topics, design thinking and data, by asking the following research question:

How could a collaborative visual tool facilitate the ideation and development process of data-oriented products and services for non data experts?

The paper is structured as follows: Section 2 discusses the related work about visual collaborative tools and canvases. Afterwards, the paper explains the research method. In the subsequent section, the paper describes our suggested concept of data thinking, formulates a definition, and provides data thinking principles. These lead over to the presentation of an 
ontology as well as its visual representation - the Data Innovation Board (DIB). Finally, the paper discusses the key findings from testing the board and presents the implications as well as the limitations of the research. The paper ends with an outlook to future work for further developing the ontology and the canvas.

\section{Related work and research gap}

Nowadays, organizations are faced with the challenge to solve more and more wicked and illstructured problems [3], which involve information systems [10, 17], product development, and service design [6, 12, 31]. Most often, required information about the problem and the context, a clear understanding on how to proceed with the problem, and a single best solution are missing $[9,20]$.

In order to gain more clarity and to work on a common action plan, teams use a variety of tools and visual artifacts to support their efforts steering through the complexity of these problems [23]. Here, different tools can be used, depending on the stage the team is in. While some tools support the team members to gain a better understanding in framing the problem, others shall help to organize clear action steps and coordinate the responsibilities within the team [3].

In the past years, several canvas-based innovation tools have been developed. The Business Model Canvas (BMC) by Osterwalder and Pigneur [27] is often cited as the quasi-standard of this type of tools. From there, many tools such as the Project Canvas [13] or the Digital Innovation Board [8] take elements or visual representations of the $\mathrm{BMC}$ and develop new tools to help teams to collaborate.

So far, only few attempts have been made to create tools that incorporate a more detailed view on data. Agrawal et al. [1] suggested an AI canvas. However, its target users are sophisticated data scientists and therefore it is not a beginner's tool. On the basis of the BMC, Hartmann et al. [15] analyzed the effect of data on all building blocks of the business model. However, they presented only a conceptual model and not a tool that enables visual collaboration. They mainly focus on the impact of data on the building blocks that are embedded in the BMC.

Specifically, when working on new data-driven products and services, the complexity of working with data as well as having team members with different levels of data literacy [32] makes it reasonable to create a visual collaboration tool that anyone can work with and people can share their knowledge on. While the data literacy of the team members often varies [32], a collaboration tool for data problems also needs to provide a framework that enables people with different levels of data literacy to communicate comfortably about the issues. Hence, data-informed collaboration tools also need to reuse established visual elements of tools such as the BMC or the Digital Innovation Board. Additionally, they need to be beginner-friendly and enable participants to contribute when starting out working with data. At the same time, such tools should also provide value for more experienced users [4]. Therefore, this paper seeks to bridge the two requirements by developing and testing a canvas for data-driven products and services.

\section{Methodology}

Avdiji et al. [3] suggested three design principles that help when creating new collaborative tools for illstructured problems: (1) Framing the ill-structured problem by developing an ontology, (2) deriving a concept map for the ontology that is the visual representation of an empty problem space, and (3) the instantiation of the visualization in a way that supports shared prototyping of the solution with sticky notes. These principles were followed during the creation process of deriving an ontology and the creation of the Data Innovation Board. The paper deals with Avdiji' et al. principles in greater detail in Section 4.

In order to test the feasibility of the created tool, we followed an action design research (ADR) approach [29]. The purpose of ADR is to study and improve artifacts by changing variables based on previously made observations and experiences. Based on literature about other visual collaboration tools and design thinking we derive data thinking design principles. These are used to create an ontology and a canvas (the Data Innovation Board). We conducted several design workshops and gathered insights via interviews, surveys, and video analysis. To improve the validity of the insights, we triangulated the different methods and sources. This approach is favorable when action research is conducted on dynamic research topics like workshop formats and gamification [16]. Between the workshops, we used the learnings from the workshops to improve the artifact. The changes are summarized in detail in Section 6.

As the central treatment method, several workshops were conducted which were analyzed through observations. However, in order to create a more holistic picture, to generate valuable user feedback, and to increase the quality of the collected data, participants were additionally asked to complete a survey involving 13 questions which were answered by all participants after the workshop. Moreover, they partake in focus group interviews that were video recorded and transcribed. The transcripts were then analyzed and statements by the participants were clustered into thematic categories. 
The workshops were conducted for the following reasons: (1) to evaluate the canvas in terms of its practicality (do people understand it?), and (2) to evaluate the canvas in terms of its usefulness (does it actually have an impact?). In total, three workshops for three different industries were conducted. Similar to design thinking workshops, the workshops were conducted with a design challenge that was loosely framing the entire workshop. These challenges were specified further during the workshop as soon as new information could be added. Table 1 outlines the setup of the three workshops with the different challenges and foci. Even though the workshops had different challenges customized for the user group, they all had common aspects like a customer-to-customer challenge that is data-related, a similar structure throughout the workshop, and visual comprehension prototypes as outcomes.

\section{Table 1: Research design of the workshops}

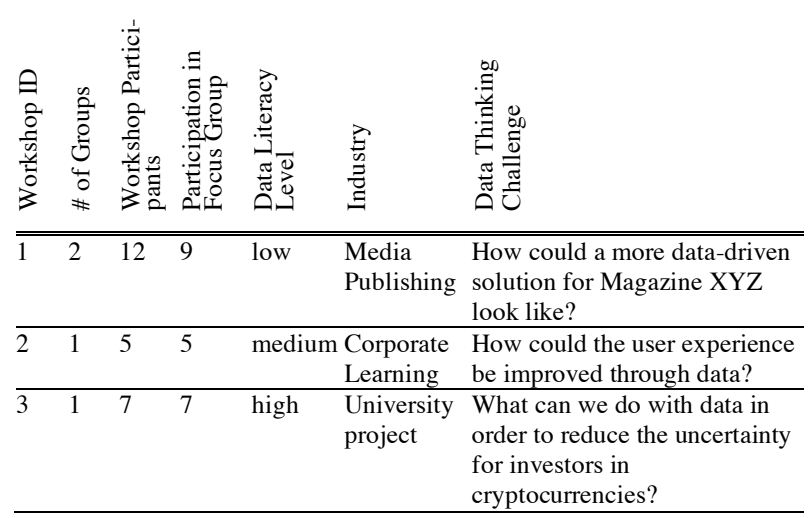

\section{Data Thinking}

In this section, we analyze the design thinking literature and abductive reasoning in order to create design principles for visual collaborative tools for datadriven design thinking projects.

From the process view, design thinking can be described as an iterative six-step process: (1) understand, (2) observe, (3) synthesis, (4) ideate, (5) prototype, and (6) test [35]. Brown [5] described the design process as a three-step process starting at a problem or opportunity space (explore), leading over to a period of brainstorming (ideate), and testing different ideas towards the implementation of the right idea (evaluate). Liedtka [22] noticed that in design thinking the user moved into the center, and empathy for the user needs became more important. The current processes "emphasize design thinking as human-centered and user-driven as a core value" [22]. Both elements are tied to the user and knowing what the user really wants. Hence, for this research, these will be placed together.
Finally, the notion of prototyping became an important part of practicing design thinking. Prototyping "is to drive real world experimentation in service to learning rather than to display, persuade, or test" [22]. Here, we define a hypothesis-based goal or destination of the prototype, but the real life examination is to change the goal or destination and/or come up with a new goal or destination entirely.

Dorst [10] showed that the fundamental reasoning pattern and the core design practices of framing and frame creation are rooted in abductive logic. According to Dorst [10], value-oriented thinking can be broken down into a formula of what, how, and value. 'What' is the subject or object. The 'how' is the working principle. Both combined create the 'aspired value' (see Figure 1).

$$
\underset{\text { (thing) }}{\text { WHAT }}+\underset{\text { (working principle) }}{\text { HOW }} \text { leads to } \quad \text { RESULT }
$$

\section{Figure 1: Pattern of abductive reasoning [10]}

In design thinking, we want to create an artifact through a working principle to create a certain value for a user or customer. The abductive reasoning pattern is going from the aspired result (value) back to possible the reasons (artifacts). Dorst [10] describes abduction as a form of productive reasoning that can be broken down into two forms. Dorst calls the first form Abduction-1. One knows the 'how' (working principle) and the 'value', but does not know the 'what'. In most cases, this is what designers would work with. They receive an aspired value, for example a design briefing, and need to design the object or service based on established working principles. In the second form of abduction (abduction-2), two variables are unknown. Only the 'value' one wants to achieve is clear. In the design thinking process, one would work most likely with abduction-2 because the 'what' and 'how', and sometimes even the aspired value are unknown at the start of a project. When it comes to data innovation projects the 'what' remains to be a product, service, or business process. However, in a data-informed design thinking ontology the 'how' is at least partly driven by data (see Figure 2). This would then be abduction-1 reasoning.

$$
\begin{gathered}
\text { ??? } \\
\text { (thing) }
\end{gathered} \underset{\text { (working principle) }}{\text { DATA leads to }} \quad \begin{gathered}
\text { VALUE } \\
\text { (aspired) }
\end{gathered}
$$

\section{Figure 2: Proposed Abduction-1 reasoning patterns with data as its working principle}

The 'what' and the 'value' remain unknown and need to be identified while going through the design thinking process. The ultimate goal, creating value through something for a potential user, would need to be 
driven by the use of data. This does not mean that other working principles, such as design and user experience, are less important.

While it is unclear from the literature how and where data is leading to value, many papers show that data "is used for the incremental improvement and optimization of current business practices and services" [15]. Moreover, Hartmann et al. [15] reported that new products and services can be disrupted by using data.

However, the challenges in a lot of data-driven innovation projects are a lack of data literacy of the participants and as a result problems in translating the technological possibilities of data into concrete ideas that create a value for the users [32]. The goal of the Data Innovation Board is to mitigate these challenges by providing a beginner-friendly visual tool for collaboratively developing data-driven products and services. The focus of the tool is on data use cases; not on concrete algorithms.

The three underlying principles: (1) user-centered design, (2) iterative prototyping including framing, and (3) abduction constitute the basis for further discussion when suggesting a data-informed design thinking methodology. Following the definition of design thinking, data thinking can be defined as a set of creative strategies that designers utilize during the process of creating new products and services where the working principle is a more detailed view on data at every single design step.

Research has shown the necessity to create integrate data-related ventures into the business model [7, 15, 24], they have yet failed to create a simple, beginner-friendly visual collaboration tool allowing users without an indepth understanding to build more data-driven business models.

Additional to the three design principles suggested by Avdiji et al. [3] (ontology, shared visualization, solution instantiation) we suggest two more design principles focusing on data-driven innovation problems: First, data as the working principle needs to be a considered part at every single step of the design process.

Data Thinking Design Principle 1: Data is the working principle of the ill-structured problem. Hence, it needs to be represented in every design step.

Second, in line with the nature of design thinking as a user-centric approach, the aspired value needs to be described based on the user.

Data Thinking Design Principle 2: The aspired value describes the value for the user which is at the center of the innovation process.
These two principles represent a first approach to derive meaningful data thinking principles and are used for deriving the ontology for the Data Innovation Board.

\section{Data Innovation Board}

The following sections describe the development of a visual collaboration tool that facilitates data thinking: the Data Innovation Board (DIB).

\subsection{Ontology}

In this section, we describe the basic ontology and explain the underlying conceptual model (Figure 3) behind the Data Innovation Board (Figure 4). Following the abduction equation, the data idea (the 'what') and the data (primary working principle) create a value for the user. Hence the Data idea, Data, and the User are predominant components when it comes to creating an ontology (Figure 3).

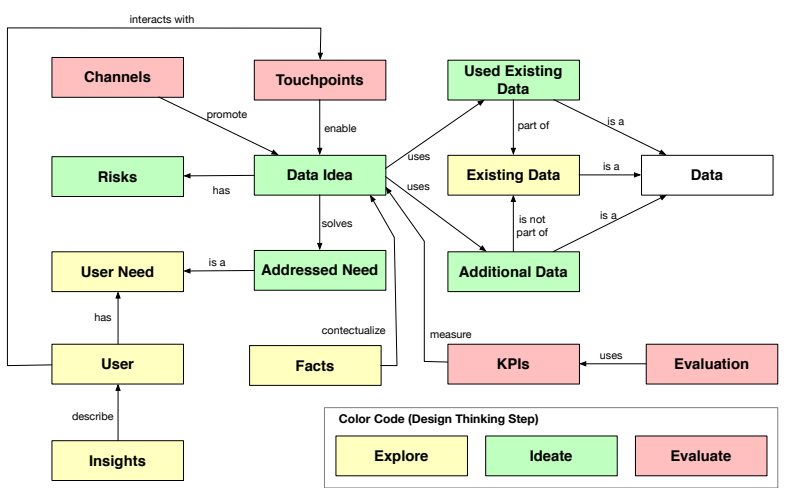

Figure 3: Ontology for the Data Innovation Board

The basic structure of the ontology follows the design thinking logic of explore, ideate, and evaluate. Hence, the first aspect the ontology must fulfill is the analysis of the status quo in a data-oriented project. The explore aspects of the ontology function rather as an asis analysis of the current situation. Here, it is important to mention that the user is explored and applicants of the board are supposed to build empathy for the user. At the same time it is necessary to draw a precise picture of the data that is already collected by an existing product or service to finalize all aspects of the as-is analysis. The ideate aspects are supposed to summarize the brainstorming period and describe the idea in more detail from the data perspective. The final evaluate aspects are elements of an action plan on how we are planning to measure the success of the idea after implementation. In the following paragraphs, the paper describes the meaning of the individual building blocks in greater detail before the final canvas is presented. 


\section{DATA INNOVATION BOARD}

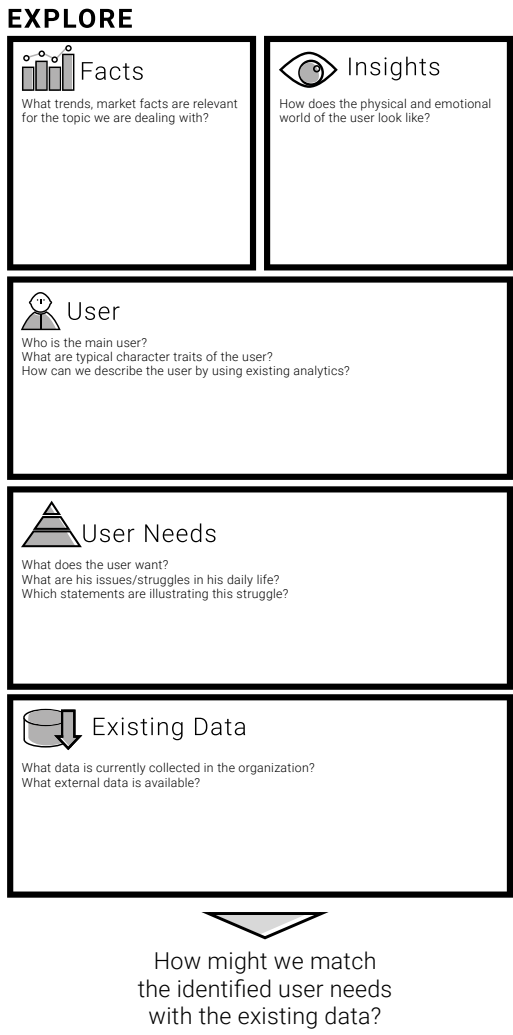

IDEATE

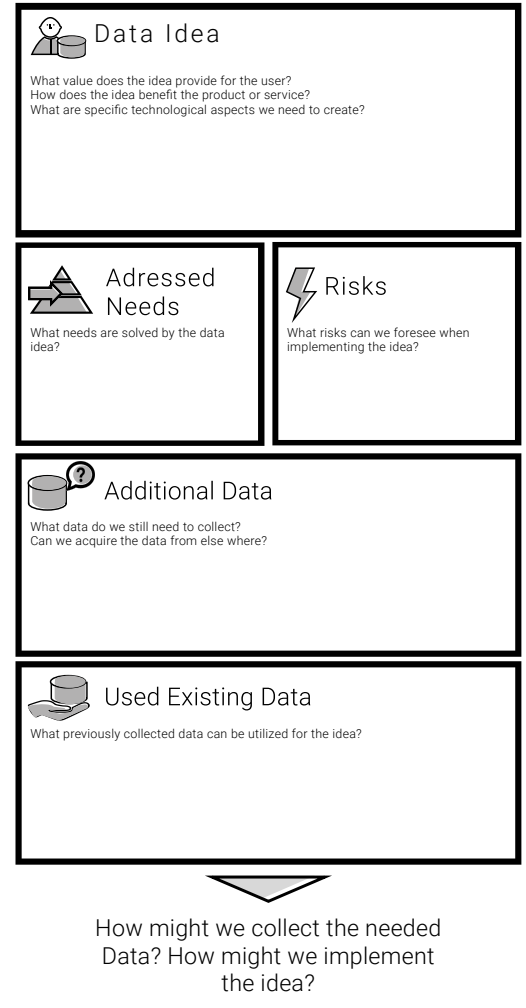

EVALUATE

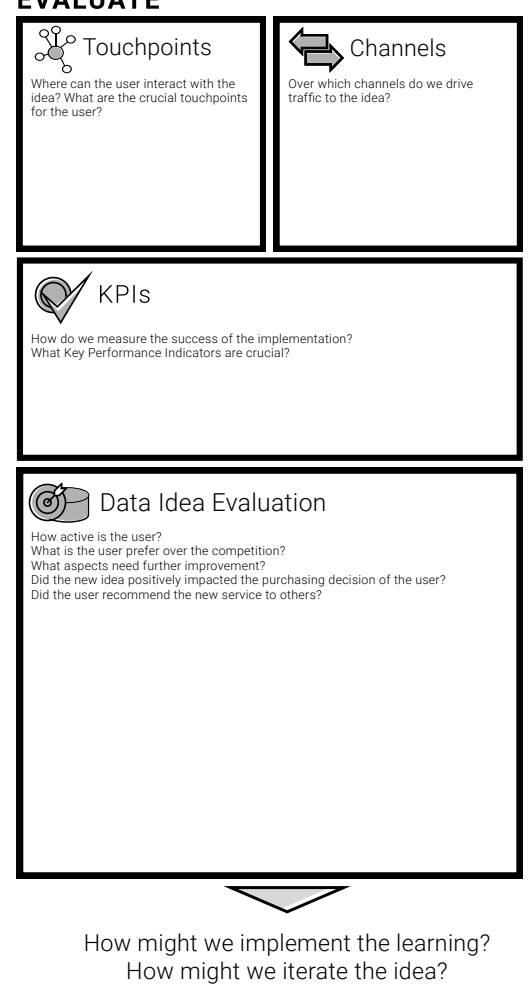

Figure 4. Data Innovation Board (DIB)

As the starting point we collect Insights about the user to better understand him or her. Facts bring further information about the general environment into the picture and contextualize the data idea. The user has certain needs that can be analyzed by conducting user research. Data is another entry point into the ontology. In the context of the DIB, Data consists of three components: Existing Data, Used Existing Data and Additional Data. Existing Data is the data that is collected at the point of time. The Data Idea uses only a fraction of the collected data (Used Existing Data). Additional Data is not part of the currently collected Existing Data but needs to be collected in order to realize the Data Idea. The Data Idea can solve a fraction of the identified User Needs. These can be addressed and isolated in Addressed Needs. However, a new Data Idea can also bring some additional risks that at the point of creation can only partially be anticipated. Those that can be anticipated fall into Risks. The idea still needs to be promoted through Channels. Channels provide the necessary traffic to raise awareness. The User interacts with the touchpoints which consequently enable the Data Idea. In order to measure the success of the Data Idea, KPIs need to be defined. The Data Idea Evaluation uses those KPIs and enriches those with qualitative observations.

\subsection{Visual canvas}

According to Avdiji et al. [3], the previously described ontology needs to be represented in a shared visualization in order to enable collaboration within teams (see Figure 4). In the classical design thinking process taught by the design schools, the design process has six distinct phases [35]. In order to reduce complexity, the DIB follows the Digital Innovation Playbook [8] and Brown [5] by only having three distinct process steps: explore, ideate, and evaluate. It follows lean principles [26, 28] and conceptually focusses on the iterative circle of build, measure, and learn. This approach is closer to what is tried to achieve with the DIB, since a new idea is supposed to be developed and tested.

In the following paragraphs, we present a new tool that incorporates the previously identified common core 
of design thinking, using established elements and trying to suggest a data-informed extension of these tools. The DIB is a visual canvas that helps teams to monitor their collaboration in terms of ideation and prototyping over a longer period of time. Hence, users of the board are able to iterate over every block and come back to it to ask whether previous assumptions were still relevant. Items defined in the blocks of the canvas can then be changed and updated based on the new insights. Hence, it follows the general design principles described in the previous section, such as starting with the user or enabling prototyping and iterations.

In order to create a framework that can be used to sharpen a more data-oriented view on new ideas, it is necessary that the current design process principles are not lost during the process. Therefore, the DIB needs to summarize the aspired value for the user, enable prototyping and iterations, and guide operators through the entire design process.

The prerequisites for a data-oriented collaboration tool are the adherence to and support of the main design principles. As such the tool needs to be user-centric, enable prototyping and still be suitable for several of iterations during the development period. In addition to this, the new concept of data needs to be included. Following the previously described ontology, the blocks are allocated in the three process steps: explore, ideate, and evaluate. At each of the three steps, linking questions connect the three different process steps. Similar to the Business Model Canvas [27], each building block has guiding questions leading the user of the tool through the board. The DIB also follows this method when asking data related questions.

In the explore area, the primary objective is to increase the understanding of the situation. In this section the team is supposed to apply divergent thinking. This means, in this step the user is explored as broad as possible. Additionally, it is required to understand the data that exists within the organization, or more specifically, what data the organization currently owns or stores about the user, its behavior and other valuable data sources. These are described in the Existing Data block.

The linking question that leads over to the ideation period is 'how might we solve the user needs with the existing data we currently have?'. The question is the introduction of the brainstorming period. In the ideate area, the Data Idea is described in more detail. The team is following a convergent thought process. It summarizes the user needs that are addressed, potential risks, as well as the data that is used and that still needs to be added additionally. Additional data can be acquired from an external source, like open data portals, or it needs to be collected internally.
The link questions that need an answer before users of the DIB can proceed are 'how might we collect the additional data?' and 'how are we planning on implementing the prototype?'.

In the third stage, the evaluate period, the main objectives are to measure the success of the idea and collect the learnings for later iterations. As such, Touchpoints enable the data idea. This is where the user is getting in contact with the new idea to receive the value. However, in most of the cases the idea needs traffic. Hence, Channels should summarize how the collaborating team is planning on promoting the new idea and from where they want to acquire traffic. All observations about the performance, new learnings about the user interaction and general experiences with the newly implemented data idea can be collected in the evaluation period. Those qualitative learnings are partly using the KPIs that measure the general performance of the data idea.

The link questions at this stage, initializing the new iteration, are 'how might we implement the learnings?' and 'how might we iterate the data idea?'.

\section{Evaluation workshops}

The workshops were conducted for two reasons: (1) to evaluate the canvas in terms of its practicality (do people understand it?), and (2) to evaluate the canvas in terms of its usefulness (does it actually have an impact?).

The workshop followed the process suggested by the canvas itself-focusing first on the exploration of the user and existing data, going over to an ideation and brainstorming period, and ending in a prototyping session. Different techniques and methods, established and known in the field of design thinking, have been applied to enable these different process steps, for example user interviews, dot voting, etc. Due to the time constraint, the workshop ended with the presentation of paper-based comprehension prototypes where participants were asked to provide information on how they are planning on implementing the idea. Hence, the participants would present also the third part of the canvas - the evaluate section.

\subsection{Key findings}

The key findings will follow the previously suggested workshop criteria: (1) the practicality of the canvas, and (2) the evaluation of the usefulness of the tool.

The practicality of the canvas was evaluated by triangulating the interviews, surveys, and video analyses. The identified problems led to a change of the canvas after each iteration of the action design research 
cycle. The reasons for the problems can be brought down to two main causes: (1) misunderstanding of the building blocks and (2) building blocks were ignored.

While Figure 4 shows the final version of the Data Innovation Board after several iteration cycles, the first layout of the board needed various adjustments. Some blocks were often questioned by the participants, which is why they were moved or renamed. For example, the first version had Touchpoints in the explore area and a bigger building block in the ideate area called User Data Artefacts, intended to summarize the idea. The evaluate area had a field called Data Goal intended to summarize the purpose and value of the new idea. Touchpoints was moved over to the evaluate area to focus the view of the workshop participants in the first stage mainly on the user and the existing data (as-is). User Data Artefacts was split into Data Value Proposition which changed to Data Idea, as it also caused confusion, and we made the differentiation between Additional Data and Used Existing Data. In order to provide a clearer guidance from one area to the other, we added the linking questions after the second workshop for the final test of the workshop. In general, participants had a lot of questions about the meaning of some of the building blocks. However, this is not very surprising because it is a new tool and for most of the participants this is a new way to think about a problem. Nevertheless, adding the guiding questions for each block of the DIB helped them formulate answers for each area. Moreover, we changed the terminology of some building blocks in later iterations to increase clarity. The Data Idea block was previously labeled as Data Value Proposition. Additionally, the two blocks Used Data and Additional Data were previously merged into one block called User Data Artefacts that caused a lot of confusion. These changes led to a clearer process and less questions by the participants. Moreover, it needs to be mentioned that the data literacy of the participants often varied a lot. In general, it can be noticed that teams with higher data literacy struggled with the classical design thinking elements, like the description of the user, while participants with a lower data literacy were facing challenges with the data-related blocks. The DIB worked best for teams where at least one participant had a high data literacy.

Another practical change to the workshop format was to conduct user interviews as a homework prior to the workshop day, as well as collecting the existing data of the organization beforehand. This had the effect that the participants were already better informed about data in general when the workshop started. Hence, it is recommendable to conduct an educational data session prior to the workshop in order to bring the general data literacy of the participants closer together.
For all workshops, $76 \%$ of the participants claimed that the workshop was clearly structured while $65 \%$ stated that it helped them comprehend the situation better.

One participant reported in the end that "the format works great for the kick-off of a project or when you do a data project, to get together as a team planning it [with the canvas]." Hence, the DIB is of help to start a new data project and to get an idea of what kind of user needs could be potentially solved with the data. All groups reported that the canvas helped them to understand the overall situation better and that they were able to understand better what data is collected within their company.

The second evaluation criterion was whether the canvas was useful and hence made an impact. First, it needs to be stated that it is not possible to evaluate the long- and mid-term effects of applying the canvas as the format and the research design didn't allow the evaluation in this regard. Instead, the research allowed to make immediate statements as well as to observe the feasibility of the idea that the participants developed during the course of the design thinking workshop. Across all workshops, participants reported that the canvas helped them to generate an overview of their idea but also to communicate with other colleagues about the necessary IT resources they would need. $81 \%$ of the participants in the workshop claimed that now they have a better understanding of what it means to build a datadriven product and service. $65 \%$ claimed that their data literacy subjectively improved and $67 \%$ claimed they would find it beneficial to continue working with the board. One participant especially noted the guidance the DIB provides:

"For me it's not that restrictive; it's rather giving guidance [...]. I actually like the progress, given the short period of time we had. I like how involved everybody got and how far we got in just a couple of hours in terms of getting a pretty holistic picture of the whole thing already. That is impressive".

More remarkable was that through the work with the canvas two groups realized that they needed to build an essential feature and hence identified a weakness in their current product. Specifically, they both wanted to build a personal online environment for their users but realized that they cannot proceed building this without collecting the necessary information as the basic product was missing a login. As a result, they designed a user journey through a login that would show the value of the personal online experience and would consequently convince the user to share her data. This example demonstrates the usefulness of the canvas for real-life projects. 


\subsection{Potential improvements}

During the course of the three workshops, several changes have been made in order to gradually improve the canvas. However, this mainly involved changes to terminologies, guiding questions in every block, and linking questions from one block to the other. These changes were positively noted by the last workshop's participants, as they provide a clearer process to which the canvas can be followed.

Partly because of the time constraints of the workshop format, the evaluate area of the canvas probably needs some further improvements. Specifically, the field of the Data Idea Evaluation can only benefit from a more detailed representation of evaluation factors of a new implemented data idea. A possible result could be that the block is split into several blocks in order to achieve a higher information quality. Additional workshops shall be conducted in order to further improve the canvas.

\section{Discussion}

The paper provides a visual canvas following a datainformed design thinking ontology. The Data Innovation Board (DIB) has been tested using an action design research approach accompanied with a quantitative survey and qualitative interviews. The DIB is of potential benefit for teams focusing on developing digital, data-driven products and services. The DIB helps users to sharpen their perspective on data challenges and to come up with a more holistic view on data in their products and services. Additionally, the tool helps practitioners to raise data awareness and improve the data literacy of participants in data thinking workshops.

Simultaneously with our study, several similar research attempts have been made to provide visual collaboration tools for AI technology or Big Data challenges $[1,15]$. However, in comparison to the DIB these tools are either for more sophisticated, tech-savvy users or they are focusing on the algorithms only and do not integrate a user view. The DIB would be more suitable to be used before any of the other canvases are applied as it is a beginner's tool that shall help to describe an idea and then start to be accompanied by other visual tools with more specific views on technology and algorithms.

Overall, the outcomes of the workshops were promising. However, working with the DIB also demonstrated that the level of understanding data among the participants varied tremendously, which has further implications.
During the course of the workshops and working with the canvas on several occasions, certain implications could be identified so far.

In general, teams with higher data literacy can create more sophisticated solutions using the canvas, while for beginners, the paper-based approach on the canvas helped them to overcome barriers related to digitalization and to start a data-driven innovation journey.

\subsection{Limitations}

Although the research followed an action design research approach and therefore has a rather experimental character, certain limitations that impacted the findings in this study need to be addressed. First, the suggested data-informed design thinking ontology is mainly derived from literature. While this in itself is not necessarily a limitation, it might be beneficial to confirm this ontology by reviewing it with other design thinking practitioners in order to further sharpen the ontology and the visual tool on every step of the design thinking process.

Moreover, some participants were very limited in their time as well. Thus, the format of the design thinking workshops needed to be tested in one working day. Normal design sprints [19] take several days. Therefore, a lot of assumptions about target users and the design challenges needed to be made by the workshop facilitator. However, due to the fact that the main goal of the workshop was to test the suitability of the suggested tools, these limitations are considered acceptable.

Furthermore, the limited number of workshops that were conducted to test the usability and impact of the canvas require more iterations of the format to further gain detailed insights on the efficacy of data-driven design thinking workshops and the applicability in different contexts. Moreover, the resulting prototypes of the data thinking workshops would need to be built and be analyzed for whether they are more data-driven than normal prototypes. The format was tested with companies that already have a product and therefore already have the target users. When continuing the tests, the format would also need to be tested with a group of participants that start out with nothing more than an idea.

Finally, one of the biggest limitations might be the subjective bias of the researcher. As it is common in action research, a large part of the analysis is based on the observations of the researcher. Thus, it is possible that the results are biased. In order to tackle this issue, the research used the triangulation of different data sources to soften these effects. 


\subsection{Future Work}

The canvas as such works as a beginner-tool for new data-oriented projects where participants have lower data literacy. However, in order to create a sound experience in the data thinking workshops further tools need to be developed. These should enable participants, for example, to brainstorm on data and provide them with the information that they need in order to describe the single building blocks of the DIB in a higher granularity. Furthermore, these tools could then follow gamification aspects that enable participants to actively track their experiences while working with data handson. In addition to that, it might be beneficial to further test the long-term application of the DIB and possibly rework and redesign some elements in the evaluate area of the canvas.

In a next research step, it seems to be necessary to analyze the usage of the collaboration tool over an entire project circle in order to gain further insights on the suitability for data-oriented, ill-structured projects.

\section{References}

[1] Agrawal, A., J. Gans, and A. Goldfarb, "A Simple Tool to Start Making Decisions with the Help of AI", Harvard Business Review Online, 2018. https://hbr.org/2018/04/asimple-tool-to-start-making-decisions-with-the-help-of-ai

[2] Atchison, S., and J. Burby, "Big data and creativity: What we can learn from 'House of Cards"', The Next Web, 2016. https://thenextweb.com/insider/2016/03/20/data-inspirescreativity/

[3] Avdiji, H., D. Elikan, S. Missonier, and Y. Pigneur, "Designing Tools for Collectively Solving Ill-Structured Problems", Proceedings of the 51st Hawaii International Conference on System Sciences (HICSS), (2018), 400-409.

[4] Bhargava, R., and C. D’Ignazio, “Designing Tools and Activities for Data Literacy Learners", (2015).

[5] Brown, T., "Design Thinking”, Harvard Business Review 86(6), 2008, pp. 84-92.

[6] Brown, T., Change by Design: How Design Thinking Transforms Organizations and Inspires Innovation, Harper Collins, New York, 2009.

[7] Chesbrough, H., and R.S. Rosenbloom, "The role of the business model in capturing value from innovation: evidence from Xerox Corporation's technology spin-off companies", Industrial and Corporate Change 11(3), 2002, pp. 529-555.

[8] Dark Horse, Digital Innovation Playbook: Das unverzichtbare Arbeitsbuch für Gründer, Macher und Manager., Murmann Publishers, Hamburg, 2016.

[9] Dorst, K., "Design Problems and Design Paradoxes", Design Issues 22(3), 2006, pp. 4-17.
[10] Dorst, K., "The core of 'design thinking' and its application”, Design Studies 32(6), 2011, pp. 521-532.

[11] Frick, W., "An Introduction to Data-Driven Decisions for Managers Who Don't Like Math", Harvard Business Review Online, 2014. https://hbr.org/2014/05/anintroduction-to-data-driven-decisions-for-managers-whodont-like-math

[12] Gruber, M., N. de Leon, G. George, and P. Thompson, "Managing by Design", Academy of Management Journal 58(1), 2015, pp. 1-7.

[13] Habermann, F., and S. Karen, Project Design: Thinking Tools for Visually Shaping New Ventures, Becota, Berlin, 2017.

[14] Harris, J., "Data Is Useless Without the Skills to Analyze It", Harvard Business Review Online, 2012. https://hbr.org/2012/09/data-is-useless-without-the-skills

[15] Hartmann, P.M., M. Zaki, N. Feldmann, and A. Neely, Big Data for Big Business? A Taxonomy of Data-driven Business Models used by Start-up Firms, University of Cambridge, Cambridge, UK, 2014.

[16] Hussein, A., "The use of Triangulation in Social Sciences Research: Can qualitative and quantitative methods be combined?", Journal of Comparitive Social Work(1), 2009, pp. 12.

[17] John, T., and D. Kundisch, "Why Fit Leads to Surprise: An Extension of Cognitive Fit Theory to Creative Problems", ICIS 2015 Proceedings, 2015, pp. 20.

[18] Kedhane, J., "A Robot May Have Written This Story”, WIRED, 2017. https://www.wired.com/2017/02/robotswrote-this-story/

[19] Knapp, J., J. Zeratsky, and B. Kowitz, Sprint: How to Solve Big Problems and Test New Ideas in Just Five Days, Simon and Schuster, New York, NY, 2016.

[20] Kogut, B., and U. Zander, "Knowledge of the Firm, Combinative Capabilities, and the Replication of Technology”, Organization Science 3(3), 1992, pp. 383-397.

[21] Leviathan, Y., and Y. Matis, "Google Duplex: An AI System for Accomplishing Real-World Tasks Over the Phone", Google AI Blog.

http://ai.googleblog.com/2018/05/duplex-ai-system-fornatural-conversation.html

[22] Liedtka, J., "Perspective: Linking Design Thinking with Innovation Outcomes through Cognitive Bias Reduction: Design Thinking", Journal of Product Innovation Management 32(6), 2015, pp. 925-938.

[23] Lundmark, L.W., J.A. Nickerson, and D. Derrick, "Wicked Problem Formulation: Models of Cognition in the Design and Selection of Valuable Strategies", Academy of Management Proceedings 2017(1), 2017, pp. 17551.

[24] Manyika, J., M. Chui, B. Brown, et al., "Big data: The next frontier for innovation, competition, and productivity McKinsey", https://www.mckinsey.com/business- 
functions/digital-mckinsey/our-insights/big-data-the-nextfrontier-for-innovation

[25] McAfee, A., and E. Brynjolfsson, "Big Data: The Management Revolution", Harvard Business Review, 2012. https://hbr.org/2012/10/big-data-the-management-revolution

[26] Mueller, R.M., and K. Thoring, "Design Thinking vs. Lean Startup: A Comparison of two User-Driven Innovation Strategies", Proceedings of the DMI 2012 International Research Conference: Leading Innovation through Design, Design Management Institute (2012), 151-161.

[27] Osterwalder, A., and Y. Pigneur, Business Model Generation: A Handbook for Visionaries, Game Changers, and Challengers, Wiley, Hoboken, NJ, 2010.

[28] Ries, E., The lean startup: How today's entrepreneurs use continuous innovation to create radically successful businesses, Crown Books, New York, 2011.

[29] Sein, Henfridsson, Purao, Rossi, and Lindgren, "Action Design Research”, MIS Quarterly 35(1), 2011, pp. 37.

[30] Sivarajah, U., M.M. Kamal, Z. Irani, and V. Weerakkody, "Critical analysis of Big Data challenges and analytical methods", Journal of Business Research 70, 2017, pp. 263-286.

[31] Steen, M.S., M.M. Manschot, and N. Koning, "Benefits of Co-design in Service Design Projects - ProQuest", International Journal of Design 5(2), 2011, pp. 53-60.

[32] Sternkopf, H., and R.M. Mueller, "Doing Good with Data: Development of a Maturity Model for Data Literacy in Non-governmental Organizations", Proceedings of the 51st Hawaii International Conference on System Sciences (HICSS), IEEE Computer Society (2018), 5045-5054.

[33] Sweney, M., "Netflix gathers detailed viewer data to guide its search for the next hit", The Guardian, 2014. http://www.theguardian.com/media/2014/feb/23/netflixviewer-data-house-of-cards

[34] Thoring, K., and R.M. Müller, "Understanding the creative mechanisms of design thinking: an evolutionary approach", Procedings of the Second Conference on Creativity and Innovation in Design, TU Delft/IASDR (2011), 137-147.

[35] Thoring, K., and R.M. Müller, "Understanding Design Thinking: A Process Model based on Method Engineering", Proceedings of the International Conference on Engineering and Product Design Education, (2011), 1-6. 